\title{
The influence of kerogen content on some brittleness indicators in a rich organic shale: comparative analysis of different approaches.
}

\author{
Oksana Bokhonok, Universidade de São Paulo, Instituto de Astronomia, Geofísica e Ciências Atmosféricas, Rua do Matão \\ 1226, 05508-090 São Paulo, SP, Brazil. oksana.bokhonok@iag.usp.br, and \\ Claudia L. Ravazzoli, CONICET and Facultad de Ciencias Astronómicas y Geofísicas, Universidad Nacional de La Plata, \\ Paseo del Bosque s/n, B1900FWA, La Plata, Argentina. claudia@fcaglp.unlp.edu.ar
}

Copyright 2016, SBGf - Sociedade Brasileira de Geofísica

Este texto foi preparado para a apresentação no VII Simpósio Brasileiro de Geofísica, Ouro Preto, 25 a 27 de outubro de 2016. Seu conteúdo foi revisado pelo Comitê Técnico do VII SimBGf, ous ño necessariamente repren Tecnico do VII SimBG, de seus associados. E proibida a reprodução total ou parcial deste material para propósitos comerciais sem prévia autorização da SBGf.

\begin{abstract}
In this work, we model some accepted brittleness indicators in rich organic shales. The rock is modeled as an effective poroelastic vertical transversely isotropic medium (VTI), composed by clay minerals and kerogen. The elastic properties of this composite medium are computed using the anisotropic theory of $\mathrm{Ciz}$ and Shapiro. By calibrating this model for the black shales of the Bakken formation (Williston basin, EEUU), we analyze the behavior and sensitivity to kerogen content of Poisson ratio, Young's modulus and its product with bulk density. Those coefficients are computed in normal (vertical) and parallel to bedding (horizontal) directions, using apparent isotropic definitions, the exact anisotropic formulation and the approximate anisotropic expressions recently derived by Thomsen for weak VTI anisotropy. While for Young's modulus we observed an excellent agreement between the different approaches, we found that Poisson ratios derived from the apparent and approximate isotropic approaches can lead to significant errors.
\end{abstract}

\section{Introduction}

Organic-rich shales have long been recognized as source rocks for clastic reservoirs, but more recently, they have gained importance as unconventional reservoirs (Vernik and Milovac, 2011). These rocks, which can be regarded as source, reservoir and seal at the same time, are generally composed by minerals (such as clay, quartz and carbonates), organic matter (kerogen) and pore fluids. The estimation of the elastic properties of those multiphase rocks is a very important subject in rockphysics, geophysics and geomechanics. The production of hydrocarbons in organic shales involves special techniques such as hydraulic fracturing, a procedure that generates tensile failure within the rocks. For a proper design of those operations as well as for other problems in geomechanics, it is essential to know the brittleness of the rocks, one of its most important properties. Although a unique definition doesn't exist, it is generally accepted that it can be estimated by means of Young's modulus, Poisson's ratio and some other attributes.

With this idea, in this work we use rock physics tools to model the elastic properties in a rich organic shale.
Following the ideas in Carcione et al. (2011), the rock is modeled as an effective poroelastic VTI medium composed by clay minerals and kerogen. The overall elastic properties are computed using the anisotropic theory of $\mathrm{Ciz}$ and Shapiro (2007). By calibrating this model for the black shales of the Bakken formation, we analyze the behavior and sensitivity to kerogen content of Young's modulus, Poisson's ratio and a related attribute defined as the multiplication of Young's modulus and bulk density. Those coefficients are computed in normal (vertical) and parallel to bedding (horizontal) directions, using apparent isotropic definitions, the exact anisotropic formulation and the approximate anisotropic expressions derived by Thomsen (2013) for weak VTI anisotropy.

\section{Rock physics model for organic shale}

It is a well established fact that black organic shales are generally characterized by strong velocity anisotropy, low velocity in the bedding-normal direction, and relatively low density and porosity (Vernik and Nur, 1992, Vernik and Liu, 1997). These rock properties are of interest in seismic attribute studies, but even more so in geomechanical applications related to reservoir characterization and hydraulic fracturing. This kind of rocks generally show VTI behavior, with axis of symmetry perpendicular to the bedding-parallel lamination and clay particle-preferred orientation (Vernik and Milovac, 2011). Understanding the relations between the bulk elastic moduli and wave velocities with parameters such as kerogen content, fluid saturation, porosity, orientation, and others, is a very important task in rock physics. This has motivated the interest of many authors resulting in the publication of different workflows to accomplish this goal.

With this in mind, and following Carcione et al. (2011), in this work we use the anisotropic poroelastic model derived by $\mathrm{Ciz}$ and Shapiro (2007), hereafter denoted as CS. They obtained the effective undrained compliance tensor (inverse of the stiffness tensor), for a porous medium in which the pore infill and solid grains are anisotropic materials. This model is the anisotropic generalization of Brown and Korringa (1975) and Gassmann's (1951) equations. The elastic properties of the dry mineral matrix are obtained using the heuristic generalization of the petroelastc model of Krief et al. (1990) for anisotropic behaviour, proposed by Carcione et al. (2011), to match the wave velocities measured by Vernik and Nur (1992). In this way, the Bakken shale is modeled as an effective VTI medium, composed by illite and kerogen in variable fractions, using parameters given by Vernik and Nur (1992) and Carcione et al. (2011). 
We remark that the model coefficients are checked to satisfy the positive definite property of the elastic strain energy. Given that the model can be extended to include more mineral phases and pore fluids (such as brine, oil and gas), such thermodynamic restriction guarantees the stability of the model for different volumetric fractions (Bokhonok and Ravazzoli, 2014).

\section{Young's modulus, Poisson's ratio and combined attribute}

It is generally accepted in the oil-gas industry that zones with relatively high Young's modulus and low Poisson's ratio are more brittle and have higher reservoir quality (Holden et al. 2013). This information is essential to find high potential areas for hydraulic fracturing and to decide the location and trajectory of the wells. As is well known, most of modern seismic studies in shale prospects are made using anisotropic data acquisition and processing tools. However, it is still common that the geomechanical analysis of these rocks, is based on isotropic parameters such as Young's modulus $E$ and Poisson's ratio $v$ (Thomsen, 2013). Despite of this theoretical inconsistency, for practical reasons seismic velocities are used to estimate vertical and horizontal Young's modulus and Poisson's ratio, using the simple relations valid for the isotropic case, hereafter referred to as apparent (Thomsen, 2013). Also, under the common condition of weak anisotropy, Thomsen (2013) found approximate expressions of the true $\mathrm{E}$ and $\mathrm{v}$ (denoted as Thomsen), as a function of vertical velocities, isotropic moduli, density and the classic anisotropy parameters (Thomsen, 1986). However, taking into account the implementation of the model described, we can make a rigorous anisotropic computation of $\mathrm{E}$ and $\mathrm{v}$, denoted as exact. This is done using the elements of the stiffness tensor given by Ciz and Shapiro (2007) for the VTI medium, and their theoretical relations with $\mathrm{E}$ and $\mathrm{v}$ in vertical and horizontal directions, as described by Thomsen (2013) and Sayers (2013). We also analyze an indicator proposed by Sharma and Chopra (2012), given by the product of Young's modulus and bulk density of the rock $\rho$, expected to be high in brittle rocks. These attributes are compared in next section using the described procedures.

\section{Comparative analysis for variable kerogen content}

Here we show model-based estimates of the mentioned attributes for variable kerogen fractions. In Figure 1a)-b), we show the variations of Young's modulus in vertical and parallel to bedding directions. A very good agreement between the three approaches can be observed, which means that reliable values of $\mathrm{E}$ can be obtained even using apparent estimations. As expected, in both directions our estimates show clearly a marked decay of these coefficients for increasing kerogen content, which implies a decrease in the brittleness of the rock. This effect can also be analyzed through the opposite behavior of Poisson's coefficients, shown in Figure 2a)-b).

In normal to bedding direction (Fig. 2a) the apparent Poisson's ratio and Thomsen's approximation show significant departures from the true exact values for almost all kerogen contents. While the apparent estimations of $v$ in parallel direction (Fig. 2b) result strongly overestimated, the Thomsen approximation
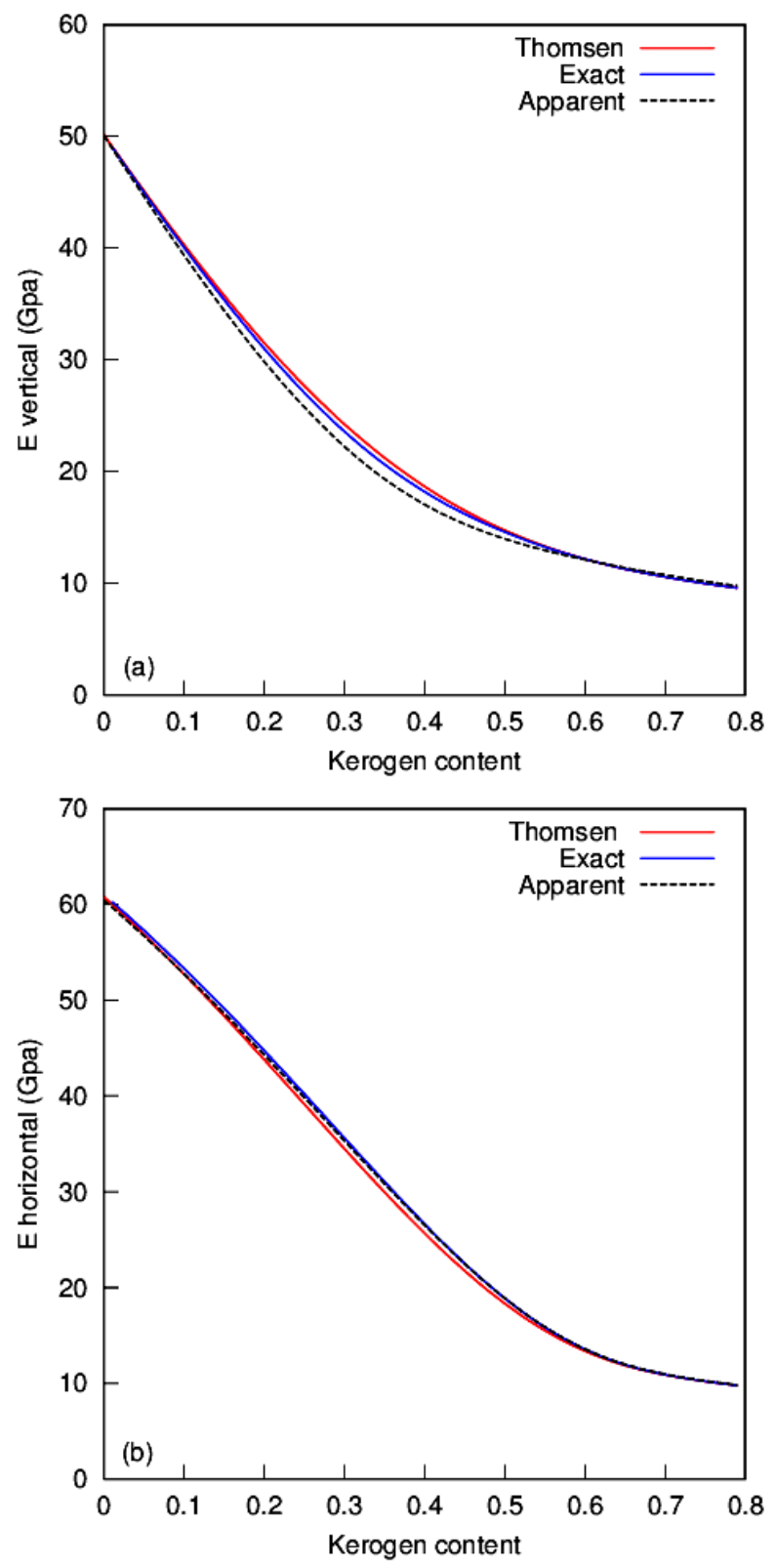

Figure 1. Young's modulus vs. kerogen content: apparent, exact and Thomsen's approximations in a) bedding-normal and b) parallel directions, derived from CS model for Bakken shale.

underestimates $v$ for almost all kerogen contents. These results indicates that Poisson's ratios derived from the isotropic approach are not good, being the horizontal estimation the least reliable of the two.

Figure 3a)-b) illustrates the combined attribute E $\rho$ vs. kerogen content. As expected, there is a very good agreement between the three computations, particularly for the normal to bedding direction, indicating that a good estimate for this attribute can be obtained from the isotropic approach. 

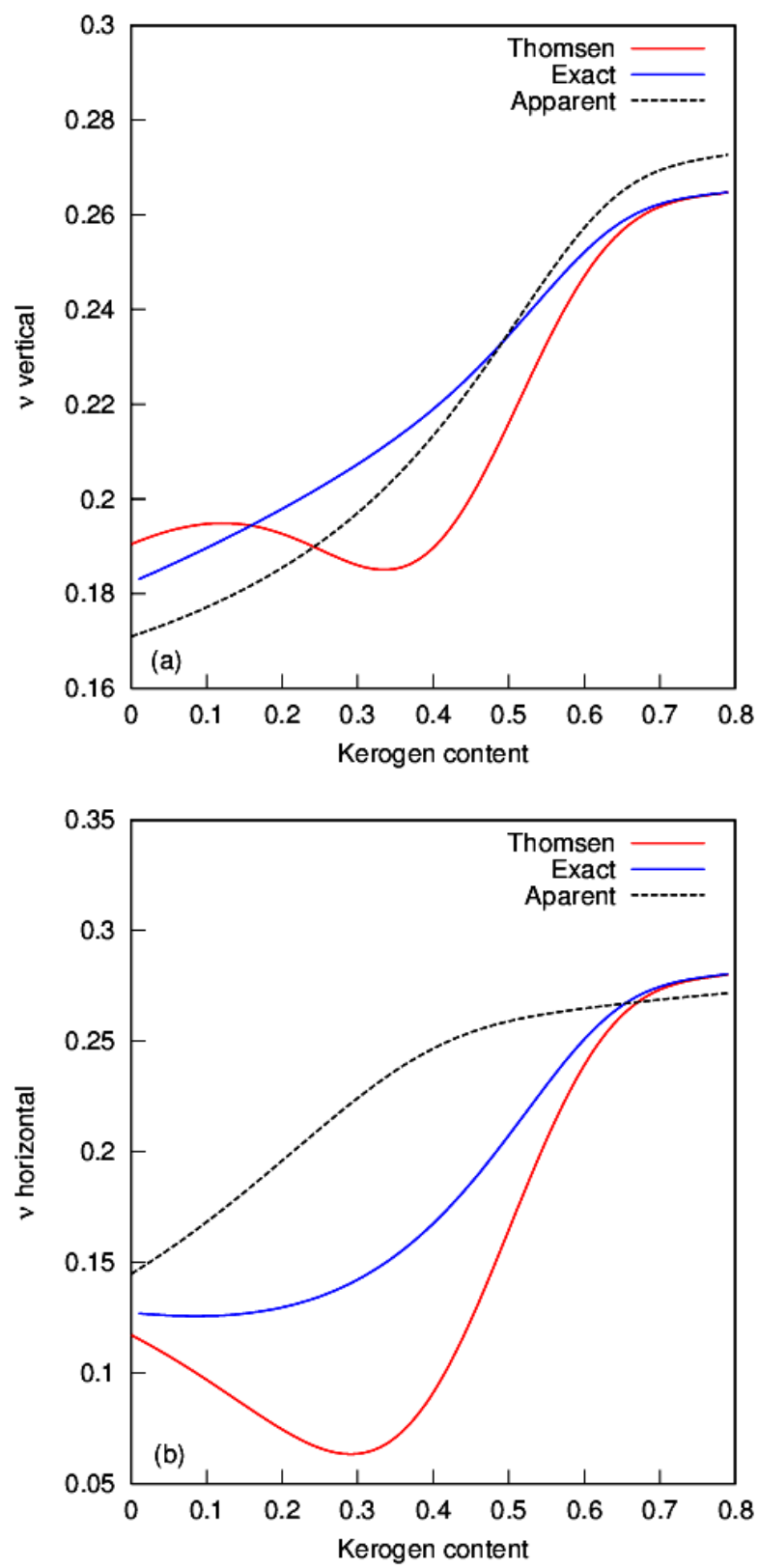

Figure 2. Poisson's ratio vs. kerogen content: apparent, exact and Thomsen's approximations in a) beddingnormal and b) parallel directions, derived from CS model for Bakken shale.

\section{Conclusions}

In this work, we implemented a rock physics model, based on the anisotropic theory of $\mathrm{Ciz}$ and Shapiro (2007), to compute brittleness indicators in a rich-organic shale. This is a topic of great interest at present due to the growing need to explore and characterize unconventional hydrocarbon resources and source rocks. Given the relevance of the kerogen content in the characterization of this kind of rocks, we used the model to compute and analyze Young's modulus, Poisson's ratio and a density-combined attribute, which are widely used as brittleness indicators, for variable kerogen fractions. The model was calibrated using information of the black
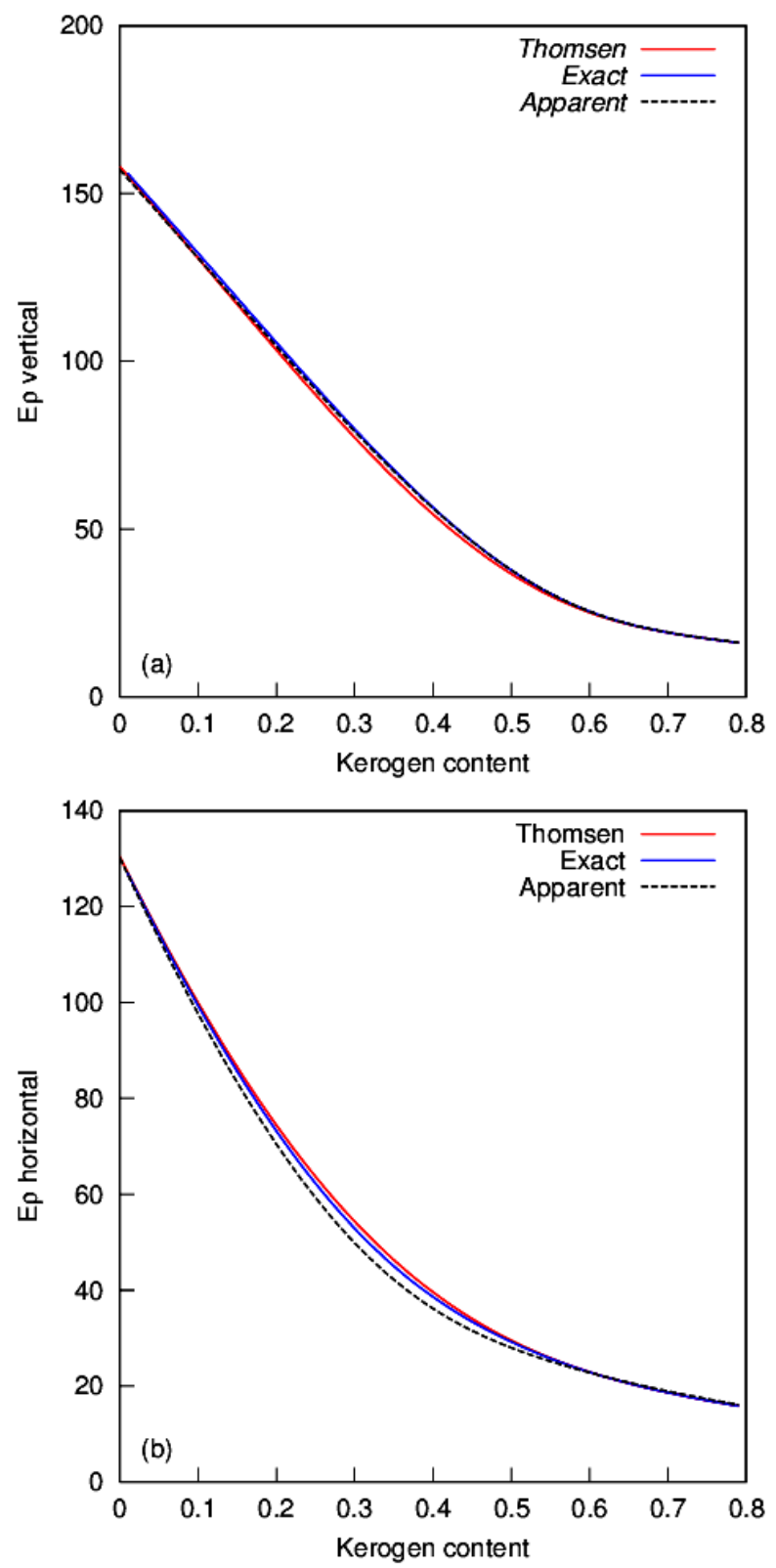

Figure 3. Combined attribute $\mathrm{E} \rho$ vs. kerogen content: apparent, exact and Thomsen's approximations in a) bedding-normal and b) parallel directions, derived from CS model for Bakken shale.

shales of the Bakken formation. For the computations, we used apparent, exact and approximate formulations. Our different model estimates show the expected decrease of brittleness with increasing kerogen content and that the rock is more brittle in parallel to bedding direction than in normal direction.

While for Young's modulus we observed an excellent agreement between the different approaches, we found that Poisson ratios derived from the apparent and approximate isotropic approaches can lead to significant errors. These discrepancies are reasonable taking into account that Poisson ratio relates lengthening in one direction to shortening in an orthogonal direction. Anyway, 
further research is needed about this subject using different rock physics models and other data sets. Nevertheless, we remark the convenience of using CS theory since it does not depend on any specific geometry for the rock constituents. Using this model the analysis can be extended to include kerogen and fluid phases such as oil, gas, brine and other mineral fractions in the inorganic rock matrix. This would be important to model the elastic properties and velocities of mature organic shales as well as to improve their geomechanical characterization.

\section{References}

Bokhonok, O. and Ravazzoli, C.L., 2014, Sensitivity study and comparative analysis of elastic properties and anisotropy coefficients in organic shales. Proceedings of E-ICES 10, International Center for Earth Sciences CDROM.Buenos Aires, November 2014.ISBN: 978-9871323-39-5.

Brown, R., and Korringa, J., 1975, On the dependence of the elastic properties of a porous rock on the compressibility of the pore fluid. Geophysics, 40, 608616.

Carcione J., Helle H. and Avseth P., 2011, Source-rock seismic-velocity models: Gassmann versus Backus. Geophysics, 76, 1-9.

Ciz, R. and Shapiro, S., 2007, Generalization of Gassmann equations for porous media saturated with a solid material. Geophysics, 72, A75-A79.

Gassmann, F., 1951, Über die elastizität poröser medien. Vier. der Natur. Gesellsch. Zürich, 96, 1-23.

Holden T., Pendrel J., Jenson F. and Mesdag P., 2013, Rock Properties for Success in Shales. Jason white papers. http://www.jason.cgg.com.

Krief, M., Garat, J., Stellingwerff, J. and Ventre, J.,1990, A petrophysical interpretation using the velocities of $P$ and $S$ waves (full-waveform sonic). Log Analyst, 31, 355-369.

Sayers C., 2013 The effect of anisotropy on the Young's and Poisson's ratios of shales.Geophysical Prospecting, 61, 416-426.

Sharma R. and Chopra S., 2012, New attribute for determination of lithology and brittleness. SEG Las Vegas 2012 Annual Meeting, Expanded Abstracts, 13891394.

Vernik, L. and Nur A., 1992, Ultrasonic velocity and anisotropy of hydro-carbon source rocks: Geophysics, 57, 727-735.

Vernik, L. and Liu X., 1997, Velocity anisotropy in shales: a petrophysical study, Geophysics, 62 (2), 521-532.

Vernik, L. and Milovac, J., 2011, Rock physics of organic shales, The Leading Edge 30 (3), 318-323.

Thomsen L., 1986, Weak elastic anisotropy, Geophysics, 51, 1954-1966.

Thomsen L., 2013, On the use of isotropic parameters $\lambda$, $\mathrm{E}, \mathrm{v}, \mathrm{K}$ to understand anisotropic shale behavior, SEG Houston 2013 Annual Meeting, Expanded Abstracts, 320324. 